\title{
New Third- and Sixth-Order Derivative-Free Techniques for Nonlinear Equations
}

\author{
F. Soleymani \& V. Hosseinabadi \\ Department of Mathematics, Zahedan Branch, Islamic Azad University, Zahedan, Iran \\ E-mail: fazl_soley_bsb@yahoo.com
}

Received: November 3, 2010 Accepted: November 19, 2010 doi:10.5539/jmr.v3n2p107

\begin{abstract}
Some local convergent derivative-free methods are suggested for solving nonlinear scalar equations. The error equations are given theoretically to show that the proposed techniques have third- and sixth-order convergence. Per cycle, the novel cubically schemes comprise three evaluations of the function while the sixth-order method includes four function evaluations. The theoretical results are supported by numerical tests to illustrate the accuracy of the contributed methods.
\end{abstract}

Keywords: Nonlinear scalar equations, Derivative-free methods, Efficiency index, Error equation, Asymptotic convergence constant

\section{Prerequisites}

Consider that the scalar function $f$ has a simple zero in the domain $D$. Finding proper approximations for the roots of nonlinear equations is in the focus of many researchers. The famous Newton's method which is given by $x_{n+1}=$ $x_{n}-f\left(x_{n}\right) / f^{\prime}\left(x_{n}\right)$, has quadratic convergence and requires one evaluation of the function and one evaluation of the first derivative per cycle. To circumvent on the derivative calculation in Newton's iteration, Steffensen first coined the following scheme

$$
x_{n+1}=x_{n}-\frac{f\left(x_{n}\right)^{2}}{f\left(x_{n}+f\left(x_{n}\right)\right)-f\left(x_{n}\right)},
$$

by replacing the derivative in Newton's iteration with forward finite difference. (1) possesses the second-order of convergence. In similar ways, many derivative-free or derivative-involved methods have already been given to literature; see e.g. (Traub, 1964) and (Soleymani, 2011).

Now in such situation at least an index; e.g. convergence rate or efficiency index, is required to evaluate the performance of different methods. The concept of efficiency index can be described as follows: an iterative process of order $p$ which comprises $n$ evaluations per iteration has the efficiency index $p^{1 / n}$ (Traub, 1964). Accordingly, Steffensen's method (1) has the efficiency index 1.414, just like Newton's method.

The main advantage of Steffensen's method is that it has quadratic convergence like Newton's method - that is, both methods find roots to the function $f$ just as quickly. But the formula for Newton's iteration requires a separate function for the derivative; Steffensen's method does not. Therefore, Steffensen's method can be programmed for a generic function.

For comparison, the Secant method needs only one function evaluation per step, thus with two function evaluations the secant method can do two steps, and two steps of the secant scheme increase the number of correct digits by a factor of 1.6. The equally time-consuming single step of Steffensen's (or Newton's) method increases the correct digits by a factor of 2 .

Generalization of iterative processes for solving system of nonlinear equations is also of grave importance. To discuss more, it is well-known that there are some close connections between finding a local minimum and solving a system of nonlinear equations. For instance, given a set of $n$ equations with $n$ unknowns; seeking a solution $x^{*}$ is equivalent to minimizing the sum of squares when the residual is zero at the minimum, hence there is a particularly close connection to the Gauss-Newton methods. In fact, the Gauss-Newton step for local minimization and the Newton step for nonlinear equations are exactly the same. Also, for a smooth function, Newton's method for local minimization is the same as Newton's method for the nonlinear scalar equations. Not surprisingly, then, many aspects of the algorithms are similar (Prentice, 2010).

As we mentioned, Newton's and Steffensen's schemes have 1.414 as the efficiency index. In practical studies, this efficiency index is not quite reliable and hence so many methods of higher orders have been proposing to the world of Numerical Analysis; see e.g. (Soleymani, 2010b).

In this work, we are concerned with simple roots of nonlinear scalar equations by iterative processes in which there are no derivative evaluation per iteration in the vicinity of the root $\alpha$. For this reason, studying multiple roots of such equation (Soleymani and Sharifi, 2010), solving system of nonlinear equations (Prentice, 2010) and derivative-involved methods 
(Saeed and Khthr, 2010) are beyond our study. Now let us review some important derivative-free methods for calculating $\alpha$.

The derivative-free method of Sidi (Sidi, 2008) of order at most two is given in the form below

$$
x_{n+1}=x_{n}-\frac{f\left(x_{n}\right)}{p_{n, k}^{\prime}\left(x_{n}\right)}, \quad n=k, k+1, \ldots
$$

whence $x_{0}, x_{1}, \ldots, x_{k}$ are initial approximations to be provided by user. And also by defining

$$
p_{n, k}(x)=f\left(x_{n}\right)+\sum_{i=1}^{k} f\left[x_{n}, x_{n-1}, \ldots, x_{n-i}\right] \Pi_{j=0}^{i-1}\left(x-x_{n-j}\right),
$$

and

$$
p_{n, k}^{\prime}(x)=f\left[x_{n}, x_{n-1}\right]+\sum_{i=2}^{k} f\left[x_{n}, x_{n-1}, \ldots, x_{n-i}\right] \Pi_{j=1}^{i-1}\left(x-x_{n-j}\right),
$$

where $f\left[x_{n}, x_{n-1}, \ldots, x_{n-i}\right]$ are divided differences of $f(x)$. This scheme is far away from a good efficiency index, because with three points per iteration (as an example case), it possesses 1.839 as the convergence rate and 1.225 as the efficiency index.

In 2007, a cubically derivative-free method (Jain, 2007) in which there is three evaluations of the function per cycle had been proposed in what follows

wherein the efficiency index is 1.442 .

$$
\left\{\begin{array}{l}
y_{n}=x_{n}-\frac{f\left(x_{n}\right)^{2}}{f\left(x_{n}+f\left(x_{n}\right)\right)-f\left(x_{n}\right)}, \\
x_{n+1}=x_{n}-\frac{f^{3}\left(x_{n}\right)}{\left[f\left(x_{n}+f\left(x_{n}\right)\right)-f\left(x_{n}\right)\right]\left[f\left(x_{n}\right)-f\left(y_{n}\right)\right]},
\end{array}\right.
$$

In 2010, Dehghan and Hajarian (Dehghan and Hajarian, 2010) suggested another cubically derivative-free scheme in the form below which also includes three evaluations of the function per iteration

$$
\left\{\begin{array}{l}
y_{n}=x_{n}-\frac{f\left(x_{n}\right)^{2}}{f\left(x_{n}+f\left(x_{n}\right)\right)-f\left(x_{n}\right)}, \\
x_{n+1}=x_{n}-\frac{f\left(x_{n}\right)\left[f\left(y_{n}\right)+f\left(x_{n}\right)\right]}{f\left(x_{n}+f\left(x_{n}\right)\right)-f\left(x_{n}\right)}
\end{array}\right.
$$

and it possesses 1.442 as the efficiency index too.

In 2011, the quartically Cordero et al. method (Cordero et al., 2011) was given in the form below which is free from derivative calculation to reach the convergence rate four while it consists of four evaluations of the function to possess 1.414 as its efficiency index

$$
\left\{\begin{array}{l}
y_{n}=x_{n}-\frac{2 f\left(x_{n}\right)^{2}}{f\left(x_{n}+f\left(x_{n}\right)\right)-f\left(x_{n}-f\left(x_{n}\right)\right)}, \\
x_{n+1}=x_{n}-\frac{2 f\left(x_{n}\right)^{2}}{f\left(x_{n}+f\left(x_{n}\right)\right)-f\left(x_{n}-f\left(x_{n}\right)\right)} \frac{f\left(y_{n}\right)-f\left(x_{n}\right)}{2 f\left(y_{n}\right)-f\left(x_{n}\right)} .
\end{array}\right.
$$

\section{Construction of new schemes}

In this paper, we seek for accurate derivative-free methods in which there are three and four evaluations of the function per iteration to possess the efficiency indices 1.442 and 1.565 respectively. A celebrated acceleration technique comprises composing two iterative methods of orders $p$ and $q$ to each other for obtaining a new method of order $p q$. Anyhow, this process increases the number of evaluations per cycle. Hence, in order to improve the efficiency index of the composed method, the idea is to introduce estimations that reduce the number of evaluations, maintaining the convergence rate as high as possible.

Let us take into consideration the one variable function $f$ to be sufficiently differentiable in the domain $D=(a, b)$. We take into account the follow-up two-step cycle

$$
\left\{\begin{array}{l}
y_{n}=x_{n}-\frac{f\left(x_{n}\right)}{f^{\prime}\left(x_{n}\right)} \\
x_{n+1}=y_{n}-\frac{f\left(y_{n}\right)}{f^{\prime}\left(y_{n}\right)}
\end{array}\right.
$$

in which there are four evaluations per iteration. It is easy to check that the scheme (8) has 1.414 as the efficiency index. To achieve our goal, we must solve two problems in the cycle (8). The problems are as follows. First, it has four evaluations per iteration and second, it includes two derivative evaluations. For these reasons, we approximate the first derivative of the function in the second step $f^{\prime}\left(y_{n}\right)$ using the method of un-determined coefficients. In this way, we consider that an estimation of $f^{\prime}\left(y_{n}\right)$ could be attained such that (8) is written in the following form

$$
\left\{\begin{array}{l}
y_{n}=x_{n}-\frac{f\left(x_{n}\right)}{f^{\prime}\left(x_{n}\right)}, \\
x_{n+1}=y_{n}-\frac{a\left(f\left(x_{n}\right)\right)^{b}+c f\left(x_{n}\right) f\left(y_{n}\right)+d\left(f\left(y_{n}\right)\right)^{e}}{g\left(f\left(x_{n}\right)\right)^{h}} \frac{f\left(y_{n}\right)}{f^{\prime}\left(x_{n}\right)} .
\end{array}\right.
$$


It is obvious that (9) requires three evaluations per cycle. But the main challenge is to obtain the unknown parameters $a, b, c, d, e, g$, and $h$ as the convergence order remains unchanged. Toward this aim, we find the Taylor expansion of (9) around the simple root $\alpha$. Hence by finding the error equation of (9), we get to know that we must select

$$
a=g=1, b=h=c=2, d=e=0,
$$

to derive the highest possible order. Now we have a simplified fourth-order method with two evaluations of the function and one evaluation of the first derivative as comes next

$$
\left\{\begin{array}{l}
y_{n}=x_{n}-\frac{f\left(x_{n}\right)}{f^{\prime}\left(x_{n}\right)} \\
x_{n+1}=x_{n}-\frac{f\left(x_{n}\right)}{f^{\prime}\left(x_{n}\right)}\left[1+\frac{f\left(y_{n}\right)}{f\left(x_{n}\right)}\left(1+2 \frac{f\left(y_{n}\right)}{f\left(x_{n}\right)}\right)\right]
\end{array}\right.
$$

Our goal is not achieved yet, because (11) is not derivative-free. To overcome on this drawback, we should estimate the first derivative $f^{\prime}\left(x_{n}\right)$ efficiently. Steffensen, Jain, Dehghan and Hajarian, and lots of other researchers have used the forward finite difference approximation to estimate $f^{\prime}\left(x_{n}\right)$ in their researches. As a consequence, the forward finite difference approximation

$$
f^{\prime}\left(x_{n}\right)=\frac{f\left(x_{n}+h\right)-f\left(x_{n}\right)}{h}+O(h)
$$

with $h=f\left(x_{n}\right)$ is taken into consideration in the cycle (11) to estimate $f^{\prime}\left(x_{n}\right)$ effectively. Therefore, we obtain the following contributed derivative-free method

$$
\left\{\begin{array}{l}
y_{n}=x_{n}-\frac{f\left(x_{n}\right)}{f\left[x_{n}, w_{n}\right]} \\
x_{n+1}=x_{n}-\frac{f\left(x_{n}\right)}{f\left[x_{n}, w_{n}\right]}\left[1+\frac{f\left(y_{n}\right)}{f\left(x_{n}\right)}\left(1+2 \frac{f\left(y_{n}\right)}{f\left(x_{n}\right)}\right)\right]
\end{array}\right.
$$

wherein $w_{n}=x_{n}+f\left(x_{n}\right), f\left[x_{n}, w_{n}\right]$ is divided differences of $f\left(x_{n}\right), f\left(w_{n}\right)=f\left(x_{n}+f\left(x_{n}\right)\right)$ and can be given by

$$
f\left[x_{n}, w_{n}\right]=\frac{f\left(w_{n}\right)-f\left(x_{n}\right)}{w_{n}-x_{n}} .
$$

The novel method (13) comprises three evaluations of the function per cycle while it is derivative-free and reaches the order of convergence three. Theorem 1 demonstrates its error equation.

Theorem 1. Let the scalar function $f$ be sufficiently smooth in the real open domain D. Further assume that $\alpha$ is its simple zero. Then the method (13) satisfies in the follow-up error equation

$$
e_{n+1}=-\frac{\left(1+c_{1}\right)}{c_{1}} c_{2}^{2} e_{n}^{3}+O\left(e_{n}^{4}\right)
$$

Proof. To demonstrate that $-\frac{\left(1+c_{1}\right)}{c_{1}} c_{2}^{2}$ is the asymptotic convergence constant of (13) wherein $c_{j}=\frac{f^{(j)}(\alpha)}{j !}, j \geq 1$, we expand any terms of (13) around the simple root $\alpha$ in the $n$th iterate. Thus, we write

$$
f\left(x_{n}\right)=c_{1} e_{n}+c_{2} e_{n}^{2}+c_{3} e_{n}^{3}+c_{4} e_{n}^{4}+O\left(e_{n}^{5}\right),
$$

and also

$$
w_{n}=x_{n}+f\left(x_{n}\right)=-\left(-1+c_{1}+e_{n}\left(c_{2}+e_{n}\left(c_{3}+c_{4} e_{n}\right)\right)\right)\left(c_{1}-c_{2} e_{n}\left(-1+c_{1}+e_{n}\left(c_{2}+e_{n}\left(c_{3}+c_{4} e_{n}\right)\right)\right) e_{n}+c_{3} e_{n}^{2}+O\left(e_{n}^{3}\right) .\right.
$$

Hence, we obtain

$$
x_{n}-\frac{f\left(x_{n}\right)^{2}}{f\left(x_{n}+f\left(x_{n}\right)\right)-f\left(x_{n}\right)}=\alpha+\left(1+\frac{1}{c_{1}}\right) c_{2} e_{n}^{2}+\frac{\left(-\left(2+\left(-2+c_{1}\right) c_{1}\right) c_{2}^{2}+c_{1}\left(1+c_{1}\right)\left(2+c_{1}\right) c_{3}\right)}{c_{1}^{2}} e_{n}^{3}+O\left(e_{n}^{4}\right) .
$$

In the same vein, we have

$$
f\left(y_{n}\right)=\left(1+c_{1}\right) c_{2} e_{n}^{2}+\left(-\frac{\left(2+c_{1}\left(2+c_{1}\right)\right) c_{2}^{2}}{c_{1}}+\left(1+c_{1}\right)\left(2+c_{1}\right) c_{3}\right) e_{n}^{3}+O\left(e_{n}^{4}\right) .
$$

Now using (18) and (19), we attain

$$
\frac{f\left(x_{n}\right)}{f\left[x_{n}, w_{n}\right]}\left[1+\frac{f\left(y_{n}\right)}{f\left(x_{n}\right)}\left(1+2 \frac{f\left(y_{n}\right)}{f\left(x_{n}\right)}\right)\right]=e_{n}-\frac{\left(1+c_{1}\right)}{c_{1}} c_{2}^{2} e_{n}^{3}+O\left(e_{n}^{4}\right) .
$$

Finally, the Taylor expansion in the last step of (13) using relation (20) gives us

$$
e_{n+1}=x_{n+1}-\alpha=-\frac{\left(1+c_{1}\right)}{c_{1}} c_{2}^{2} e_{n}^{3}+O\left(e_{n}^{4}\right)
$$


which shows that method (13) is of order three with only three evaluations of the function per iteration. As a consequence, the efficiency index of (13) is 1.442 which is bigger than 1.225 of the example case of (2), 1.414 of (1), (7) and Newton's iteration, and is equal to 1.442 of (5) and (6). This completes the proof.

It is remarked that for attaining an optimal two-step method with three evaluations, the convergence rate should be 4 . And the order of (13) is one unit lower. We also pull the attention towards this fact that, one can replace (12) with backward finite difference. If we consider a more complicated variant of backward finite difference for approximating $f^{\prime}\left(x_{n}\right)$ as follows

$$
f^{\prime}\left(x_{n}\right) \approx \frac{f\left(x_{n}\right)-f\left(x_{n}-\theta f\left(x_{n}\right)\right)}{\theta f\left(x_{n}\right)},
$$

then by assuming $f\left(w_{n}\right)=f\left(x_{n}-\theta f\left(x_{n}\right)\right)$, another novel family of third-order methods could be constructed as comes next

$$
\left\{\begin{array}{l}
y_{n}=x_{n}-\frac{f\left(x_{n}\right)}{f\left[x_{n}, w_{n}\right]}, \\
x_{n+1}=x_{n}-\frac{f\left(x_{n}\right)}{f\left[x_{n}, w_{n}\right]}\left[1+\frac{f\left(y_{n}\right)}{f\left(x_{n}\right)}\left(1+2 \frac{f\left(y_{n}\right)}{f\left(x_{n}\right)}\right)\right]
\end{array}\right.
$$

where $\theta \in R-\{0\}$ and its error equation is $e_{n+1}=x_{n+1}-\alpha=\frac{\left(1-c_{1} \theta\right) \theta c_{2}^{2}}{c_{1}} e_{n}^{3}+O\left(e_{n}^{4}\right)$. Experimental results show that for very small values of $\theta$ the attained numerical results are very good.

Now we can achieve more convergence rate and index of efficiency by developing (13). In order to obtain a sixth-order derivative-free method with better efficiency index, we perform a new Newton's iteration after the second step of (13). Clearly, the attained method has five evaluations per full cycle to reach the convergence rate 6 and due to this, that method is inefficient. At this moment, the main challenge is to replace the new-appeared first derivative of the function at the third step such that the convergence rate does not fall down. To do this, we consider the following three-step iteration in which there are only four functional evaluations per cycle. Theorem 2 is given to demonstrate its sixth-order convergence

$$
\left\{\begin{array}{l}
y_{n}=x_{n}-\frac{f\left(x_{n}\right)}{f\left[x_{n}, w_{n}\right]}, \\
z_{n}=x_{n}-\frac{f\left(x_{n}\right)}{f\left[x_{n}, w_{n}\right]}\left[1+\frac{f\left(y_{n}\right)}{f\left(x_{n}\right)}\left(1+2 \frac{f\left(y_{n}\right)}{f\left(x_{n}\right)}\right)\right], \\
x_{n+1}=z_{n}-\frac{f\left(z_{n}\right)}{f\left[y_{n}, z_{n}\right]}\left(1-\frac{1+f\left[x_{n}, w_{n}\right]}{f\left[x_{n}, w_{n}\right]} \frac{f\left(z_{n}\right)}{f\left(w_{n}\right)}\right),
\end{array}\right.
$$

where $f\left[y_{n}, z_{n}\right]=\frac{f\left(z_{n}\right)-f\left(y_{n}\right)}{z_{n}-y_{n}}, f\left[x_{n}, w_{n}\right]=\frac{f\left(w_{n}\right)-f\left(x_{n}\right)}{w_{n}-x_{n}}$ and $w_{n}=x_{n}+f\left(x_{n}\right)$. Note that in fact (24) is attained by composition of some already known facts, i.e. an approximation of the first derivative and the weight function to gain the sixth-order convergence.

Theorem 2. Let the scalar function $f$ be sufficiently smooth in the real open domain D. Further assume that $\alpha$ is its simple zero. Then the method (24) is a sixth-order derivative-free method that satisfies in the follow-up error equation

$$
e_{n+1}=x_{n+1}-\alpha=\frac{\left(1+c_{1}\right)^{2} c_{2}^{3}\left(-\left(5+c_{1}\left(11+4 c_{1}\right)\right) c_{2}^{2}+c_{1}\left(1+c_{1}\left(3+c_{1}\right)\right) c_{3}\right)}{c_{1}^{5}} e_{n}^{6}+O\left(e_{n}^{7}\right)
$$

Proof. Same assumptions and symbolic calculations as done in the proof of Theorem 1, results in relations (16)-(21). Thus, it is needed to go on by providing the Taylor Series expansion of $f\left(z_{n}\right)$ around the solution. Hence, we have $f\left(z_{n}\right)=-\left(1+c_{1}\right) c_{2}^{2} e_{n}^{3}+\left(\left(\left(5+c_{1}\left(15+c_{1}\left(13+4 c_{1}\right)\right)\right) c_{2}^{3}-c_{1}\left(1+c_{1}\right)\left(1+c_{1}\left(5+2 c_{1}\right)\right) c_{2} c_{3}\right) e_{n}^{4}\right) / c_{1}^{2}+\left(1 /\left(c_{1}^{3}\right)\right)\left(-\left(36+c_{1}(99+\right.\right.$ $\left.\left.\left.c_{1}\left(99+c_{1}\left(46+9 c_{1}\right)\right)\right)\right) c_{2}^{4}+c_{1}\left(32+c_{1}\left(105+2 c_{1}\left(59+6 c_{1}\left(5+c_{1}\right)\right)\right)\right) c_{2}^{2} c_{3}-c_{1}^{2}\left(1+c_{1}\right)\left(2+c_{1}\right)\left(1+c_{1}\left(3+c_{1}\right)\right) c_{3}^{2}\right) e_{n}^{5}+\left(1 /\left(c_{1}^{4}\right)\right)((1+$ $\left.c_{1}\right)\left(170+c_{1}\left(313+c_{1}\left(243+c_{1}\left(95+16 c_{1}\right)\right)\right)\right) c_{2}^{5}-c_{1}\left(262+c_{1}\left(824+c_{1}\left(1027+2 c_{1}\left(334+9 c_{1}\left(13+2 c_{1}\right)\right)\right)\right) c_{2}^{3} c_{3}+c_{1}^{2}(66+\right.$ $\left.\left.c_{1}\left(239+c_{1}\left(329+c_{1}\left(230+c_{1}\left(83+12 c_{1}\right)\right)\right)\right)\right) c_{2} c_{3}^{2}\right) e_{n}^{6}+O\left(e_{n}^{7}\right)$.

Now using this relation, we obtain $f\left[y_{n}, z_{n}\right]=c_{1}+\left(\left(1+c_{1}\right) c_{2}^{2} e_{n}^{2}\right) / c_{1}+\left(\left(-\left(2+c_{1}\left(3+2 c_{1}\right)\right) c_{2}^{3}+c_{1}\left(1+c_{1}\right)\left(2+c_{1}\right) c_{2} c_{3}\right) e_{n}^{3}\right) / c_{1}^{2}+$ $\left(\left(\left(1+c_{1}\right)\left(9+c_{1}\left(11+5 c_{1}\right)\right) c_{2}^{4}-c_{1}\left(7+c_{1}\left(14+c_{1}\left(13+4 c_{1}\right)\right)\right) c_{2}^{2} c_{3}\right) e_{n}^{4}\right) / c_{1}^{3}+O\left(e_{n}^{5}\right)$.

Furthermore, we have $1-\frac{1+f\left[x_{n}, w_{n}\right]}{f\left[x_{n}, w_{n}\right]} \frac{f\left(z_{n}\right)}{f\left(w_{n}\right)}=1+\left(\left(1+c_{1}\right) c_{2}^{2} e_{n}^{2}\right) / c_{1}^{2}+\left(\left(-\left(5+c_{1}\left(18+c_{1}\left(17+5 c_{1}\right)\right)\right) c_{2}^{3}+c_{1}\left(1+c_{1}\right) 1+c_{1}(5+\right.\right.$ $\left.\left.\left.\left.2 c_{1}\right)\right) c_{2} c_{3}\right) e_{n}^{3}\right) / c_{1}^{4}+\left(1 /\left(c_{1}^{5}\right)\right)\left(\left(51+2 c_{1}\left(78+c_{1}\left(82+c_{1}\left(38+7 c_{1}\right)\right)\right)\right) c_{2}^{4}-c_{1}\left(35+c_{1}\left(128+c_{1}\left(152+c_{1}\left(77+15 c_{1}\right)\right)\right)\right) c_{2}^{2} c_{3}+\right.$ $\left.c_{1}^{2}\left(1+c_{1}\right)\left(2+c_{1}\right)\left(1+c_{1}\left(3+c_{1}\right)\right) c_{3}^{2}\right) e_{n}^{4}+1 / c_{1}^{6}\left(-\left(313+c_{1}\left(956+c_{1}\left(1135+3 c_{1}\left(229+c_{1}\left(73+10 c_{1}\right)\right)\right)\right)\right) c_{2}^{5}+c_{1}\left(385+c_{1}(1311+\right.\right.$ $\left.\left.c_{1}\left(1699+c_{1}\left(1111+c_{1}\left(381+56 c_{1}\right)\right)\right)\right)\right) c_{2}^{3} c_{3}-c_{1}^{2}\left(76+c_{1}\left(295+c_{1}\left(421+5 c_{1}\left(59+3 c_{1}\left(7+c_{1}\right)\right)\right)\right) c_{2} c_{3}^{2}\right) e_{n}^{5}+O\left(e_{n}^{6}\right)$.

Therefore, using the obtained relations, we attain

$$
e_{n+1}=x_{n+1}-\alpha=\frac{\left(1+c_{1}\right)^{2} c_{2}^{3}\left(-\left(5+c_{1}\left(11+4 c_{1}\right)\right) c_{2}^{2}+c_{1}\left(1+c_{1}\left(3+c_{1}\right)\right) c_{3}\right)}{c_{1}^{5}} e_{n}^{6}+O\left(e_{n}^{7}\right),
$$

which shows that (24) is a sixth-order derivative-free method, consuming four functional evaluations per full iteration to reach the index of efficiency 1.565 . This ends the proof. 
Note that if one chooses backward finite difference approximation as done in (22) instead of forward finite difference in (24), then a novel family of sixth-order derivative-free methods will be attained, but with a different weight function. Due to similarity, we do not bring this case into separate formulations.

\section{Numerical implementations}

The practical utilities of (13), (23), and (24) are given by solving a couple of numerical examples and comparison with other well-known methods of different orders in this section. Scientific computations in many areas of science and engineering demand high-order root solvers. We have used the second-order method of Steffensen (SM2), the third-order technique of Jain (JM3), the third-order scheme of Dehghan and Hajarian (DHM3), the fourth-order scheme of Cordero et al. (CM4) and our novel contributed techniques $(\mathbf{S H 3})=(13),(\mathbf{S H 3}(2))=(23)$ with $\theta=1,(\mathbf{S H 6})=(24)$. The test functions and their roots are given as follows:

$$
\begin{aligned}
& f_{1}(x)=\sqrt{x^{4}+8} \sin \left(\frac{\pi}{x^{2}+2}\right)+\frac{x^{3}}{x^{4}+1}-\sqrt{6}+\frac{8}{17}, \quad \alpha=-2, \\
& f_{2}(x)=\sqrt{x^{2}+2 x+5}-2 \sin (x)-x^{2}+3, \quad \alpha \approx 2.331967655883964 \text {, } \\
& f_{3}(x)=\sin ^{-1}\left(x^{2}-1\right)-\frac{x}{2}+1, \quad \alpha \approx 0.594810968398369, \\
& f_{4}(x)=x^{5}+23 x-6, \quad \alpha \approx 0.260817090224163 \text {, } \\
& f_{5}(x)=(1+\cos x)\left(e^{x}-2\right), \quad \alpha \approx 0.693147180559945 \text {. }
\end{aligned}
$$

The results are summarized in Table 1 , in terms of required number of iterations to obtain an approximation of the root which is correct up to 15 decimal places. In our numerical comparisons we have used the stopping criterion $\left|x_{n}\right| \leq 10^{-15}$. As Table 1 manifests, our contributed methods are efficient in contrast to the other high-order schemes in terms of required number of iterations. Note that method of Cordero et al. includes four function evaluations per cycle while (13) and (23) consist of three evaluations per iteration. We have used "Div." when the iteration is divergence for the considered starting point.

Thus from Table 1 and above analysis in Section 2, we can see that the new proposed methods are free from any derivative evaluation of the function $f$ in computing process. And subsequently, the new iterative formulas can be used as an alternative to the existing methods or in some cases where existing methods are not successful. Our remark is concerned with an important problem appearing in practical application of multi-point methods. As emphasized in (Traub, 1964), a fast convergence, one of the advantages of multi-point methods, can be attained only if starting points are sufficiently close to the sought roots; otherwise, it is not possible to realize the expected convergence speed in practice. For this reason, in applying multi-point root-finding methods, a special attention should be paid to finding suitable starting points.

\section{Concluding remarks}

Multi-point iterative methods for solving nonlinear equations were extensively studied in Traub (1964). This class of root solvers is of grave importance since it circumvents the theoretical limits of one-point methods regarding the convergence rate and index of efficiency. It is worth mentioning that the interest for multi-point methods has renewed in the first decade of the 21 st century. In this article, we have attained simple and elegant methods of orders three and six by using additional evaluations of the function at the point iterated by Steffensen's method for solving nonlinear equations. Thus, one requires three evaluations of the function $f$ per full step to gain the efficiency index 1.442 as well as four functional evaluations per full cycle to gain 1.565 as the efficiency index. The superiority of the presented derivative-free schemes was also corroborated by numerical results displayed in the Table 1. Finally, we conclude that the methods presented in this paper are some efficient derivative-free methods.

\section{Acknowledgments}

We are thankful to the reviewer for his/her constructive remarks and suggestions which have enhanced the present paper.

\section{References}

Cordero, A., Hueso, J.L., Martinez, E., Torregrosa, J.R. (2011). Steffensen type methods for solving nonlinear equations. Journal of Computational and Applied Mathematics, doi:10.1016/j.cam.2010.08.043.

Dehghan, M., Hajarian, M. (2010). Some derivative free quadratic and cubic convergence iterative formulas for solving nonlinear equations. Computational and Applied Mathematics, 29, 19-31.

Jain, P. (2007). Steffensen type methods for solving non-linear equations. Applied Mathematics and Computation, 194, 527-533.

Prentice, J.S.C. (2010). First-principles derivation of a third-order method for solving a two-dimensional nonlinear system. Journal of Mathematics Research, 2, 57-63.

Saeed, R.K., Khthr, F.W. (2010). Three new iterative methods for solving nonlinear equations. Australian Journal of Basic and Applied Sciences, 4(6), 1022-1030.

Sidi, A. (2008). Generalization of the secant method for nonlinear equations. Applied Mathematics E-Notes, 8, $115-123$. 
Soleymani, F. (2011). Revisit of Jarratt method for solving nonlinear equations. Numerical Algorithms, doi: 10.1007/s11075010-9433-6.

Soleymani, F., Sharifi, M. (2010). A simple but efficient multiple zero-finder for solving nonlinear equations. Far East Journal of Mathematical Sciences (FJMS), 42, 153-160.

Soleymani, F. (2010)b. Concerning some sixth-order iterative methods for finding the simple roots of nonlinear equations. Bulletin of Mathematical Analysis and Applications, 2, 146-151.

Traub, J.F. (1964). Iterative Methods for the Solution of Equations, Prentice-Hall, Englewood Cliffs, New Jersey.

Table 1. Comparison of different derivative-free methods in terms of required number of iterations

\begin{tabular}{lllllllll}
\hline Functions & Starting Points & SM2 & JM3 & DH3 & CM4 & SH3 & SH3(2) & SH6 \\
\hline$f_{1}(x)$ & -2.75 & 4 & 3 & 3 & 3 & 3 & 3 & 2 \\
$f_{1}(x)$ & -0.6 & 5 & 4 & 4 & Div. & 3 & Div. & 3 \\
$f_{2}(x)$ & 3.9 & 7 & 4 & 4 & 3 & 5 & 4 & 3 \\
$f_{2}(x)$ & -1 & 6 & 4 & 4 & Div. & 4 & 5 & 3 \\
$f_{3}(x)$ & 0.85 & 5 & 3 & 3 & 3 & 3 & 3 & 2 \\
$f_{3}(x)$ & 0.3 & 5 & 3 & 3 & 3 & 3 & 2 & 2 \\
$f_{4}(x)$ & 0.4 & 8 & 3 & 5 & Div. & 4 & 3 & 2 \\
$f_{4}(x)$ & 0.5 & 24 & 3 & 14 & Div. & 9 & 5 & 2 \\
$f_{5}(x)$ & 0.9 & 5 & 3 & 3 & 3 & 3 & 3 & 2 \\
$f_{5}(x)$ & 0.5 & 5 & 3 & 4 & 3 & 3 & 3 & 2 \\
\hline
\end{tabular}

